\title{
Talento humano como estrategia de crecimiento organizacional, caso: Empresa Comercializadora Mexicana
}

\section{The management of the human capital as a growth strategy, case Mexican Commercializing Company}

Dr. Margarita Ramírez Torres,

Universidad Autónoma de Baja California,

México

mramirez@uabc.edu.mx
Dr. Cruz Estrada,

Universidad Autónoma de Baja California,

México

icruz@uabc.edu.mx
Dr. Ana María Miranda Zavala,

Universidad Autónoma de Baja California,

México

amiranda@uabc.edu.mx

\section{RESUMEN:}

El capital humano puede diferenciar a una empresa de otra, le agrega valor y apoya su expansión. La presente investigación tiene como objetivo identificar los aspectos que influyen en la gestión del capital humano y contribuyen al crecimiento hacia nuevos mercados de la empresa comercializadora mexicana. El diseño de la investigación fue cuantitativo, no experimental y transversal. Los resultados revelan que el personal se encuentra preparado, con experiencia y habilidades para apoyar a la empresa en su etapa de crecimiento. En definitiva, uno de los factores que pueden llevar al éxito o al fracaso es el capital humano porque son las personas quienes toman las decisiones de la empresa.

\section{ABSTRACT:}

Human capital can make a difference between a company and another as it adds value to it and supports its development. This research aims at identifying the aspects that have an influence on human capital management and contribute to the growth towards new markets of the Mexican marketing company. The research design was quantitative, not experimental and transversal. The results reveal that the personnel are prepared, experienced and skilled to support the company during its growth stage. In the end, one of the factors that may lead to success or failure is human capital because it is the people who take decisions in the company.

\section{RESUMO:}

O capital humano pode ser o diferencial de uma empresa para outra, proporcionalhe valor e apoia a sua expansão. A presente investigação tem como objetivo identificar os aspectos que influem na gestão do capital humano e contribuem para o crescimento focados em novos mercados da empresa mexicana de comercialização. O desenho da investigação foi quantitativo, não experimental e transversal. Os resultados revelam que o quadro de funcionários se encontra preparado, com experiência e habilidades para apoiar o ensino na sua etapa de crescimento. Certamente, um dos fatores responsáveis pelo êxito ou pelo fracasso de uma empresa é o capital humano porque são as pessoas as quais tomam as decisões da empresa.

\section{RÉSUMÉ:}

Le capital humain peut faire la différence entre une entreprise et une autre, il luiajoute de la valeur et soutient son développement. Cette recherche vise à identifier les aspects qui influencent la gestion du capital humain et contribuent à la croissance vers de nouveaux marchés de l'entreprise commerciale mexicaine. La conception de la recherche a été quantitative et non pas expérimentale et transversale. Les résultats révèlent que le personnel est préparé, expérimenté et habilité pour soutenir l'entreprise dans son étape de développement. En définitive, un des facteurs qui peuvent mener au succès ou à l'échec est le capital humain parce que ce sont les personnes qui prennent les décisions dans l'entreprise.

\section{PALABRAS CLAVES \\ COMPAÑÍA, ADMINISTRACIÓN, COLABORADORES, ESTRATEGIAS, AMPLIACIÓN DE MERCADO.}

\section{KEYWORDS}

COMPANY ADMINISTRATION, COLLABORATORS, STRATEGIES, MARKET EXPANSION.

\section{PALAVRAS CHAVES \\ MPRESA, ADMINISTRAÇÃO, COLABORADORES, ESTRATÉGIAS, EXPANSÃO DE MERCADO}

\author{
MOTS CLÉS \\ COMPAGNIE, \\ ADMINISTRATION, \\ COLLABORATEURS, \\ STRATÉGIES, DÉVELOPPEMENT \\ DE MARCHÉ.
}




\section{INTRODUCCIÓN}

En México, la brecha digital en el sector empresarial se encuentra en aumento; según el Instituto Nacional de Estadística y Geografía (2015), las micro, pequeñas y medianas empresas (pymes) representan el 99,8 \% del total de las empresas en el país, generan el $42 \%$ del producto interno bruto y el $78 \%$ del empleo. De acuerdo con Lanzagorta (2011), publicado por el periódico El Economista, solo 2 de cada 10 pymes sobreviven más allá de dos años, debido principalmente a la falta de capacitación y profesionalismo del capital humano, así como a una inadecuada administración del negocio y una carente incorporación y uso de las tecnologías de la información y la comunicación.

Los problemas dentro de las pymes constituyen un campo muy abierto y complejo de información; no todas las empresas son iguales, pero sí comparten ciertas características. Todas las empresas tienen distintos retos, debido a que son de diferentes giros y venden diversos productos, pero la mayoría de las pymes mueren por problemas de administración y por la falta de conocimiento del factor humano (Cirer, 2008). Uno de los principales problemas con que se enfrentan las empresas en la actualidad es el desconocimiento del potencial de sus colaboradores, lo cual puede suponer una condición limitante para el desarrollo y crecimiento de la empresa. La falta de planeación y gestión del capital humano influye en la quiebra de las empresas, pues generalmente se contrata a personal sin medir la consecuencia que esto pueda generar (Ramírez, Texis y Aguilar, 2014).

La gestión del Capital humano, entonces, se convierte en un aspecto crucial: si el éxito de las pymes depende en gran medida de lo que las personas hacen y cómo lo hacen, invertir en las personas puede generar grandes beneficios. Ante este escenario, las empresas hoy deben conocer la importancia de llevar una buena gestión del capital humano.

La presente investigación se realizó en una compañía comercializadora de insumos para la industria. Es una empresa constituida bajo el régimen de persona moral, formada bajo una sociedad anónima de capital variable. Inició sus operaciones en el año 2009, tiene presencia en alrededor de treinta ciudades, destacando Baja California, Sonora, Sinaloa, Torreón, Guadalajara, Zona del Bajío, Veracruz, Estado de México, Hidalgo y Puebla, entre otras, y atiende a más de doscientos clientes a nivel local y nacional.

Su mercado objetivo son las compañías que fabrican tarimas nuevas o recicladas, localizadas en la región noroeste y del Bajío de la República Mexicana; con una capacidad de consumo mínima de treinta cajas mensuales.

Los principales productos que comercializa son pistolas neumáticas, refacciones para las pistolas, clavos para fabricar tarimas, clavillos, grapas para la industria mueblera, engrapadoras, material de empaque, flejes de polipropileno y poliéster, sellos, tensionadoras, rejas de acero, postes, abrazaderas, seguetas para cortar madera, sierras de cintas, balancines y accesorios, entre otros.

La empresa se encuentra en evolución hacia nuevos mercados; por ello, la presente investigación tiene como objetivo identificar los aspectos que influyen en la gestión del capital humano y que contribuyen al crecimiento de la empresa. Con este fin, se aplicó un cuestionario a los 24 colaboradores, de los cuales 18 corresponden a la ciudad de Tijuana y Mexicali, y 6 se encuentran laborando en Guadalajara y Bajío.

De acuerdo con Martínez (2005), la aplicación de una buena administración está relacionada con la productividad de la empresa y la simplificación del trabajo al establecer principios, métodos y procedimientos para lograr mayor eficiencia y eficacia. Por su parte, Deeb (2013) señala que una mala administración puede ejercer un gran impacto en las operaciones generales de una compañía y sus empleados. Pese a los consejos de estos dos autores, muchas empresas no analizan su fase administrativa, no reparan en las debilidades que puedan tener internamente con la gestión del capital humano y, por consiguiente, fracasan en sus proyectos de desarrollo y crecimiento organizacional.

Las carencias en la administración han conducido a las organizaciones a cerrar sus puertas de forma permanente y el pésimo liderazgo genera un mayor reemplazo de empleados; los gastos de reunir y capacitar individuos se vuelven inaccesibles, lo que cual impacta en la capacidad de una empresa para continuar con sus operaciones (Deeb, 2013). Si bien, para poder competir con otras empresas del mismo giro, una clave fundamental es el mejoramiento de su 
administración (Cirer, 2008), una buena gestión del capital humano permite crear una ventaja competitiva en el mercado, mejorar la productividad y garantizar el éxito a largo plazo.

\section{DESARROLLO}

\section{Conceptualización de la gestión de capital humano}

De acuerdo con Villalobos Monroy y Pedroza Flores (2009), la teoría del capital humano fue desarrollada por el economista estadounidense Gary Becker en 1964, quien define al capital humano como el conjunto de las capacidades productivas que un individuo adquiere por acumulación de conocimientos generales o específicos. La principal aportación que hizo Gary Becker al concepto de capital humano fue considerarlo como el mayor tesoro que podía poseer cualquier organización, pues cada individuo tiene conocimientos y habilidades que lo forman como persona única y la calidad de sus hábitos de trabajo logra aumentar la productividad de la empresa (Alonso, 2012).

Por su parte, Del Canto (2011) nos menciona que el capital humano es el recurso más importante que tiene una empresa y es necesario estudiar la manera de gestionarlo para lograr su asociación con las estrategias organizacionales que conlleven al mejoramiento de la empresa, al incremento del aprendizaje y a la eficiencia y competitividad organizacional. Es importante mencionar que un recurso es algo que se usa y se trata de maximizar su uso; cuando una persona es vista como capital humano, se trata de aumentar sus talentos por medio de cursos y capacitaciones que la preparen mejor, la actualicen y la mantengan laborando dentro de la empresa, creando valor para esta y para ellas mismas. Los seres humanos no son propiedad de la organización; las personas de una compañía son colaboradores y ofrecen sus habilidades y su tiempo laboral, mientras que la empresa remunera por ello.

La gestión del Capital humano en las organizaciones es la función que permite la colaboración eficaz de las personas (empleados, funcionarios, participantes, recursos humanos o cualquier denominación utilizada) para alcanzar los objetivos organizacionales e individuales. Los colaboradores de la empresa pueden aumentar o disminuir las fortalezas y debilidades de una organización dependiendo de la manera en que se les trate; pueden ofrecer conocimiento que a su vez crea mejoras en los productos y servicios, los cuales generan valor desde la perspectiva del cliente; y también podrían definir estrategias más eficientes para el logro de los objetivos (optimización de la maquinaria, el dinero, las instalaciones y los sistemas computacionales, entre otros) (Lev Baruch, 2001, citado en Benavides, 2012; Calderón y Mousalli, 2012).

\section{Aspectos que influyen en la gestión del capital humano}

La administración del capital humano es el proceso cuya finalidad es lograr un mejor desempeño, aprovechamiento, acrecentamiento y mejora en las capacidades, habilidades, experiencias, conocimientos y competencias del personal, es decir, la gestión de los procesos humanos y organizacionales. Con el fin de establecer un clima organizacional óptimo que contribuya a mejorar la satisfacción de las personas que se encuentran en una empresa y con ello mejorar la productividad, calidad y consecuentemente el crecimiento e innovación de la organización (Montilla, 2003; Chiavenato, 2007).

De acuerdo con Chiavenato (2007) y Domínguez (2008), para lograr una buena gestión del capital humano se recomienda desarrollar seis aspectos (ver figura 1). 


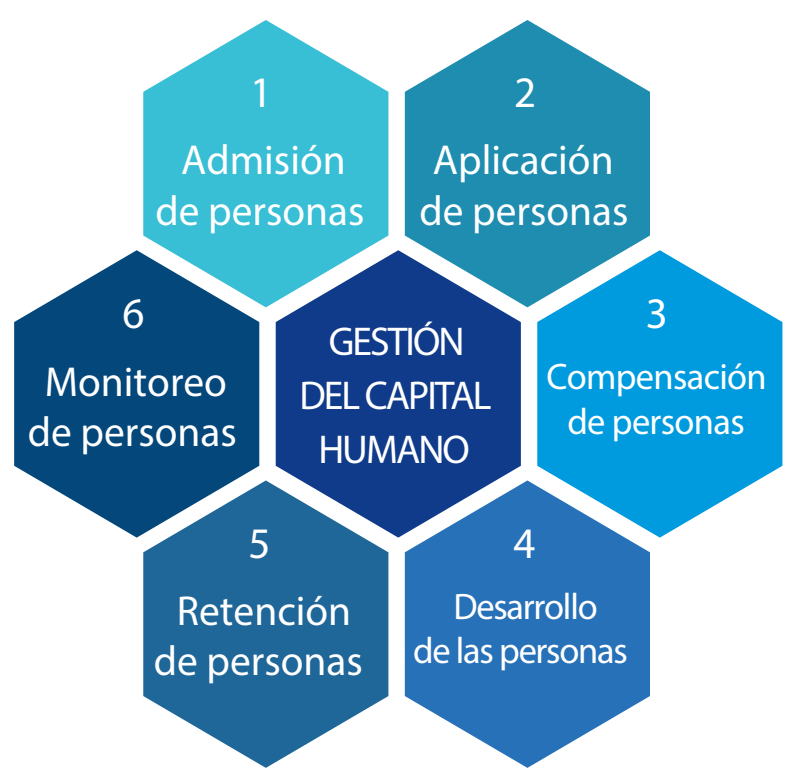

Figura 1. Aspectos que influyen en la gestión del Capital humano.

Fuente: Elaboración propia.

La selección de personal es un procedimiento para seleccionar entre los candidatos reclutados a los más adecuados, para ocupar los cargos existentes en la empresa, tratando de mantener o aumentar la eficiencia y el rendimiento del personal. Ninguna organización puede garantizar la permanencia de sus empleados; según Navarro (2005), las empresas que invierten en la formación y desarrollo de su personal tienen un índice de rotación inferior a las que no lo hacen, por lo que disponer de colaboradores motivados es la clave para la empresa.

Por su parte, la evaluación del desempeño constituye el proceso por el cual se estima el rendimiento global del colaborador. La mayor parte de los colaboradores procuran obtener retroalimentación sobre la manera en que cumplen los objetivos organizacionales para decidir las acciones que deben tomar. De acuerdo Reyes y García (2009), el mejoramiento continuo de la gestión del capital humano, es decir, su constante evaluación del desempeño, el escuchar y prestar atención a sus inconformidades y sugerencias, puede lograr una mejor eficiencia en la organización, así como la optimización de tiempos, procesos y actividades para el logro de los objetivos.

De acuerdo con la Organización Internacional del Trabajo (2016), compañías a nivel mundial se están preocupando por optimizar sus procesos para elevar su productividad y se están dando cuenta de que el éxito depende del capital humano. El mercado laboral de hoy se define por la demanda de colaboradores cada vez más preparados, con más competencias y que se adapten a nuevas estructuras de trabajo. Sin embargo, ese Capital humano también exige condiciones diferentes a las empresas. En muchos casos, los empleados están interesados en disfrutar horarios flexibles y capacitación continua, así como tener una rutina laboral clara y precisa que permita un equilibrio entre la vida profesional y la personal. 


\section{Beneficios de la gestión del capital humano}

De acuerdo con Huertas (2012), el esfuerzo humano resulta vital para el funcionamiento de cualquier organización; si el elemento humano está dispuesto a proporcionar su esfuerzo, la organización marchará; en caso contrario, se detendrá. Por este motivo, toda organización debe prestar atención a su personal primordialmente, ya que es el pilar del crecimiento organizacional. Falestchi (2013) menciona que el crecimiento es el aumento en la participación en el mercado, el número de trabajadores o bien el tamaño de las instalaciones. Supone que el crecimiento está conectado con el desarrollo corporativo, es decir: el crecimiento de una empresa es la expansión de la tarea en general, lo cual provocará más crecimiento que, a su vez, se traducirá en una mayor expansión en el trabajador. No obstante, lo mejor que se puede hacer para lograr el crecimiento de los colaboradores es alentar, facilitar el desarrollo de sus competencias, motivar, valorar los esfuerzos y los conocimientos puestos en práctica e incentivar sus ideas.

El conjunto de habilidades y capacidades del capital humano y la calidad de los servicios es el principal valor añadido para el cliente, por lo que Muratalla, Gaona, García y Guzmán (2016) aseveran que la competencia del mercado radica principalmente en la calidad de los servicios prestados por cada empresa.

Por su parte, Álvarez, Fraiz \& Del Rio (2013) aseveran que los beneficios que se presentan con una buena gestión del capital humano son los siguientes: mejoras en la relación entre colaboradores y dirección, incremento de la motivación, mejoras en la comunicación interna, incremento del compromiso, mayor productividad y apertura de nuevos mercados. Estos beneficios se pueden implementar por medio de un programa de mejora continua.

Implantar un programa de mejora continua fortalece la calidad de los servicios de la empresa ofrecidos a los clientes. Para ello, es necesario realizar un diagnóstico del capital humano que colabora en la empresa, tomando en consideración las capacidades para desempeñar las tareas laborales, mediante la gestión del conocimiento del capital intelectual y del aprendizaje organizacional, en los cuales el factor humano ocupa un lugar central y protagónico, creando sistemas de aprendizaje y desarrollo tecnológico para dirigir y potenciar el desarrollo de competencias a través del trabajo coordinado.

Así, también en el diagnóstico inicial es posible identificar los requerimientos futuros del capital humano para diseñar estrategias que lleven a satisfacer esas necesidades, partiendo de la situación actual de la empresa y permitiendo mejorar las capacidades y habilidades de los empleados actuales, con el fin de aumentar la productividad y la capacidad para resolver problemas y reaccionar de manera flexible a las exigencias cambiantes e inconstantes del ambiente (Pérez, 2012). Asimismo, es preciso crear más oportunidades de venta en otras zonas o mercados en donde exista una necesidad de sus productos o servicios, es decir, una ampliación de mercado.

\section{METODOLOGÍA}

El desarrollo de la investigación inició con la revisión literaria que permitiera conceptualizar e identificar los aspectos que influyen en la gestión del capital humano y que contribuyen al crecimiento de la empresa hacia nuevos mercados. Posteriormente, se conformó el marco teórico. Es una investigación cuantitativa, no experimental y transversal.

De acuerdo con Kerlinger y Lee (2002), en una investigación cuantitativa no experimental es imposible manipular las variables o asignar aleatoriamente a los participantes. Por su parte, Hernández, Fernández \& Baptista (2006) mencionan que el período de tiempo en que se desarrolla la investigación puede ser de tipo longitudinal o transversal. Los diseños de investigación transversal recolectan datos en un solo momento, en un tiempo único.

Por tal motivo, la metodología que se utilizó es de tipo cuantitativa, no experimental y transversal, porque se quiere conocer lo que piensan los colaboradores sobre los aspectos que influyen en la gestión del capital humano y su relación con la etapa de crecimiento organizacional. Se utilizó la encuesta como técnica de investigación.

El cuestionario se aplicó directamente a los participantes localizados en las sucursales de Tijuana, Mexicali, Guadalajara 
y Bajío en México, cuenta con preguntas en escala de Likert, dicotómicas y de opción múltiple.

El cuestionario que se utilizó en esta investigación, está conformado por las siguientes dimensiones (Tabla 1):

\begin{tabular}{l|c|c}
\multicolumn{2}{c}{ Tabla de dimensiones del cuestionario } \\
Dimensiones & No. de preguntas & Total \\
\hline Fuerza de trabajo & $1-5$ & 5 \\
\hline Conocimiento & $6-9$ & 5 \\
\hline Barreras de crecimiento & $10-13$ & 4 \\
\hline Adaptabilidad & $14-19$ & 6 \\
\hline Datos del trabajador & $20-24$ & 4 \\
\hline
\end{tabular}

Tabla 1. Tabla de dimensiones del cuestionario

Fuente: Elaboración propia.

Las dimensiones de la encuesta aplicada buscan obtener información acerca de las habilidades, capacidades y conocimiento de las funciones que desempeñan los colaboradores, y conocer la percepción del capital humano acerca del ambiente de trabajo y la administración, así como los aspectos que consideran como barreras para el crecimiento personal y organizacional, mediante una serie de preguntas entre las cuales se encuentran, entre otras, las siguientes: ¿Considera que están definidas las funciones para cada uno de los departamentos de la empresa? Dentro de las actividades que efectúan, ¿cuál considera que tiene mayor dificultad para realizarse? ¿La empresa cuenta con manual de procedimientos en donde puede guiarse para la realización de sus actividades? ¿Qué mejora desearía que se aplicara en la empresa para lograr un crecimiento hacia nuevos mercados?

Una vez terminado el levantamiento del censo, se utilizó para el análisis de los datos el SPSS Statistics.

\section{RESULTADOS}

Se analizó de manera individual cada una de las dimensiones y los ítems que integran el censo y se generaron gráficas de los resultados para una interpretación comprensible. A continuación, se ofrecen los resultados obtenidos de la encuesta aplicada a 24 personas que laboran en la empresa comercializadora.

Se inicia con las variables sociodemográficas, las cuales indican que el $83 \%$ pertenecen al género masculino y un $17 \%$ al femenino. Con relación a las edades de los colaboradores, un $39 \%$ se encuentra entre los 39 a 43 años, seguido por las personas de 29 a 33 años con un $31 \%$; los rangos de 19 a 23 y 34 a 38 años tienen ambos 9 \% y también empatan con un $4 \%$ los de 24 a 28,49 a 53 y 54 a 58. 
En lo correspondiente a los años de antigüedad en la empresa, el $74 \%$ tiene menos de tres años de laborar en ella, un $22 \%$ de los colaboradores tiene de 4 a 7 años y, finalmente, un $4 \%$ lleva de 8 a 11 años.

Por su parte, el nivel académico de los encuestados arroja que el 43 \% concluyó la educación superior, un 35 \% terminó sus estudios de educación media y un 22 \% cursó estudios de educación básica.

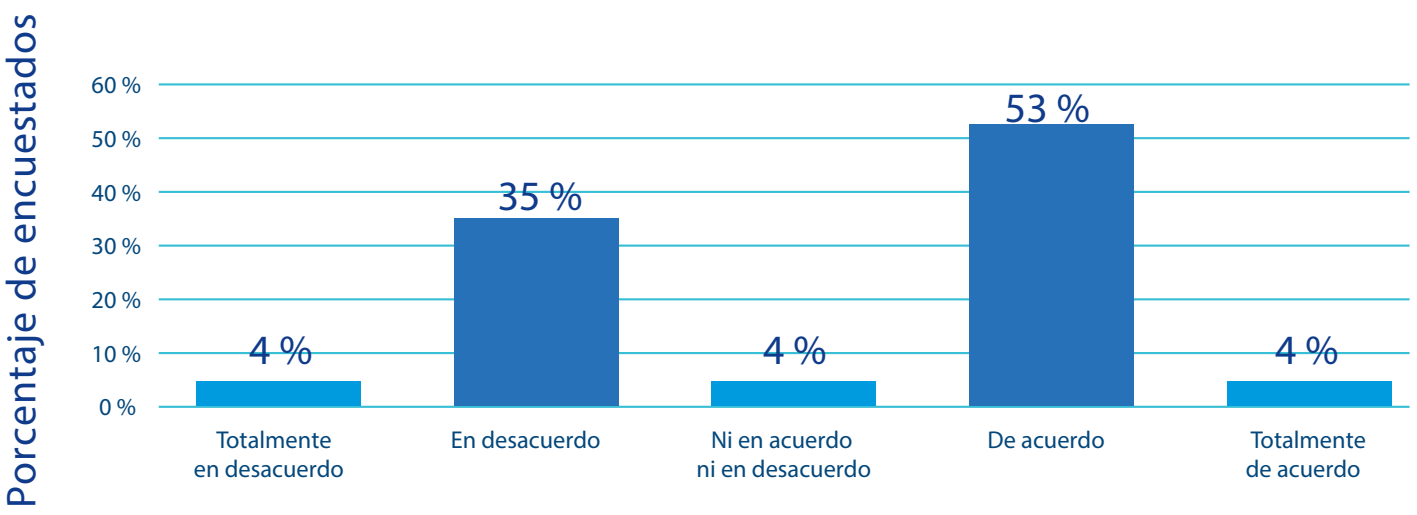

Figura 1. Las actividades que realiza en su puesto de trabajo son entendibles. Fuente: Elaboración propia.

Con respecto a las dimensiones del cuestionario, se observa en la Figura 1 que el 53 \% de los encuestados señaló estar de acuerdo con la claridad en la descripción del puesto de trabajo, el $35 \%$ indicó estar en desacuerdo y con un $4 \%$ se encuentran empatadas las opciones totalmente en desacuerdo y totalmente de acuerdo.

Con respecto a la pregunta de si cuenta con la experiencia requerida para ayudar a la empresa en su crecimiento, el $78 \%$ de los encuestados estuvo de acuerdo y el $13 \%$ se manifestó totalmente de acuerdo. También se les pregunto si consideraban tener la habilidad requerida para ayudar a la empresa en su crecimiento; los resultados arrojaron que el $79 \%$ se encuentra de acuerdo y el $14 \%$ se manifestó totalmente de acuerdo.

Sobre la definición de las funciones departamentales, en la Figura 2 se observa que un $48 \%$ se muestra en desacuerdo y un $43 \%$ de los encuestados afirma estar de acuerdo en que los puestos de trabajo para cada departamento están definidos. 


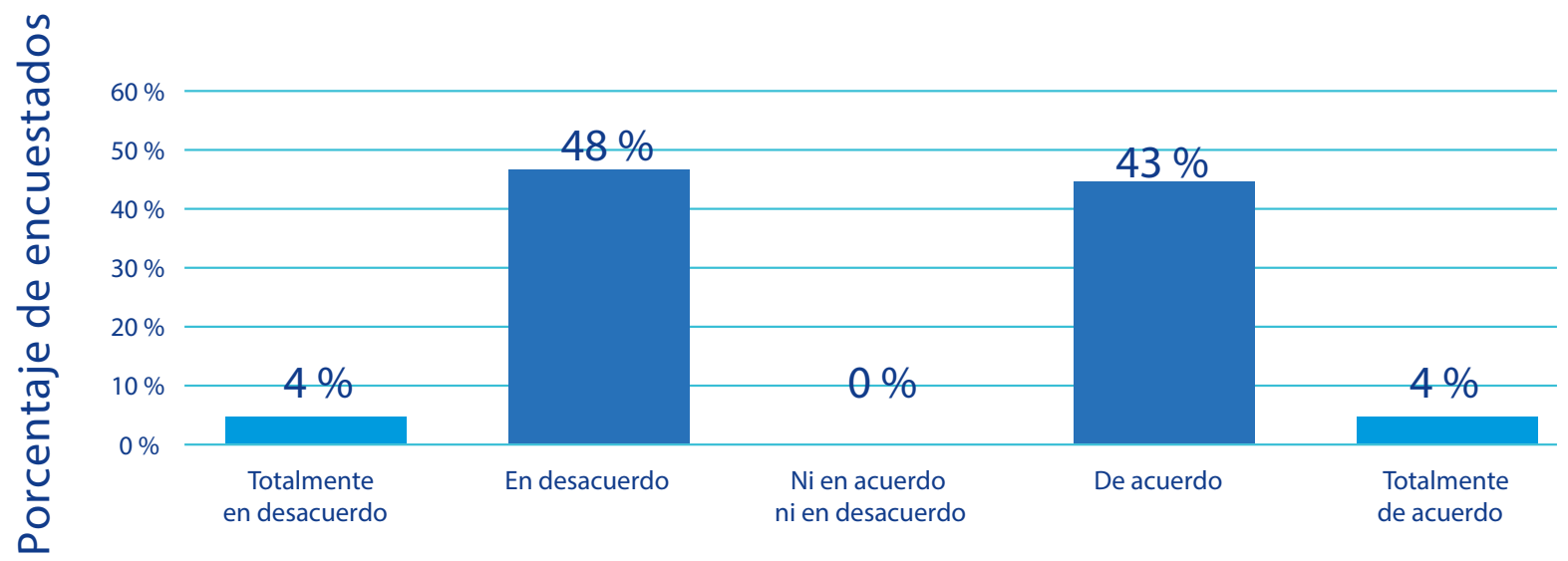

Figura 2. Están definidas las funciones para cada uno de los departamentos de la empresa. Fuente: Elaboración propia.

A la pregunta, si la empresa cuenta con manual de procedimientos con el que puedan guiarse para la realización de sus actividades, el 61 \% de los colaboradores encuestados mencionó estar en desacuerdo y, con un empate del $17 \%$, otros encuestados se manifestaron totalmente en desacuerdo y neutrales.

Sin embargo, un 91 \% del capital humano contestó que sería sencillo adaptarse a nuevos procedimientos dentro de la empresa, el $4 \%$ se inclinó por la opción muy sencilla y el $5 \%$ consideró ser poco sencillo.

En lo correspondiente a la pregunta de si recibe capacitación para realizar sus actividades, en la Figura 3 se observa que un $52 \%$ de los encuestados se expresaron en desacuerdo, un $26 \%$ indicó estar de acuerdo en que recibe entrenamiento y el $13 \%$ se mostró neutral. 


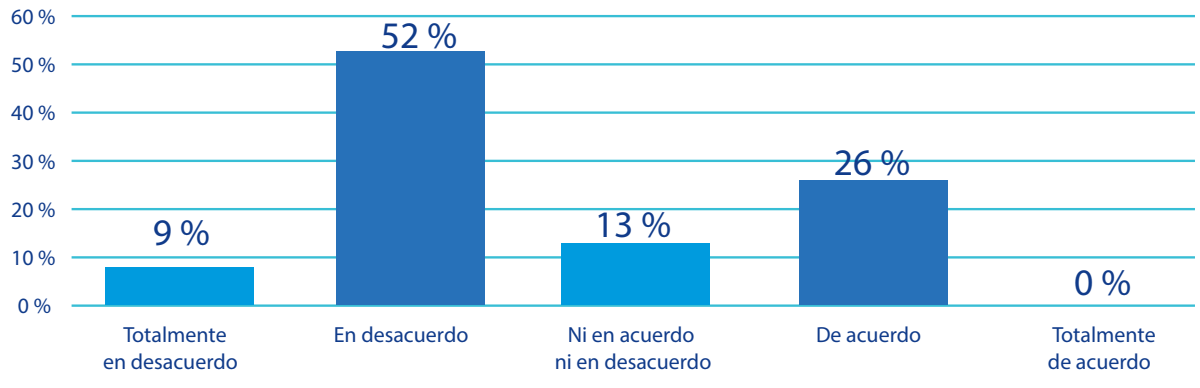

Figura 3. Recibe capacitación para el desempeño de sus funciones. Fuente: Elaboración propia.

A la interrogante, si considera que el ambiente de trabajo es óptimo para el desempeño de sus actividades, el $65 \%$ de los colaboradores estuvo de acuerdo, el $17 \%$ se manifestó en desacuerdo y un $10 \%$ neutral.

La Figura 4 muestra que el $61 \%$ de los encuestados considera que existe un departamento que paraliza el crecimiento de la empresa; sin embargo, el 39 \% contrasta con la opinión anterior.

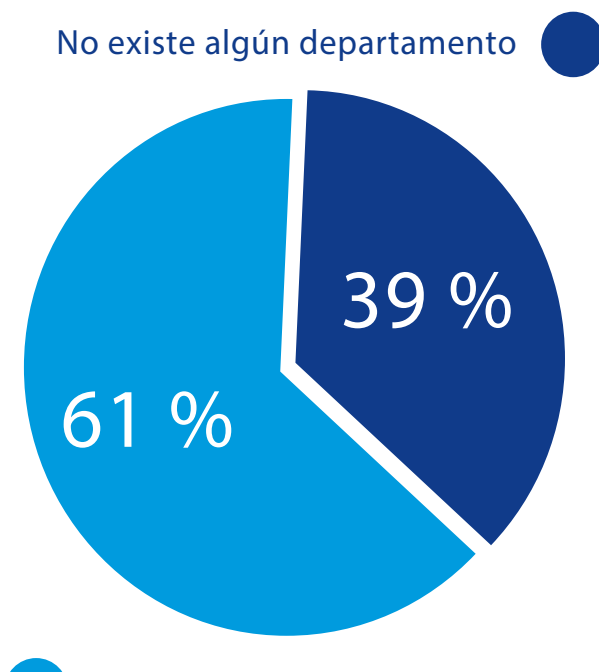

Si existe algúnde partamento

Figura 4. Existencia de un departamento que detiene el crecimiento de la empresa. Fuente: Elaboración propia. 
El área que más afecta al crecimiento de la empresa es el departamento de logística, con un 30 \%, seguido por el departamento de cobranza, con un $13 \%$, y, finalmente, con el $9 \%$, el departamento de jardinería.

Las causas que sobresalen son la mala planeación, con un $22 \%$, la falta de profesionalismo y organización, ambas con el $15 \%$, y, por último, con un $8 \%$, los motivos son las deficiencias en los procesos y comunicación organizacional. Sin embargo, el $91 \%$ de los colaboradores se siente preparado para adaptarse al rápido crecimiento y expansión de la empresa.

Con relación a la duda de si existe el trabajo en equipo, el 39 \% del capital humano confirma que las labores diarias se desarrollan en equipo, mientras que un $35 \%$ afirma que es imposible trabajar en equipo; las opiniones de los trabajadores se encuentran totalmente divididas en este tema. Sin embargo, un $74 \%$ considera tener la experiencia y las habilidades necesarias para ayudar a la organización en su etapa de crecimiento hacia nuevos mercados.

\section{CONCLUSIONES}

Después del análisis de los resultados obtenidos en esta investigación, se concluye que la empresa cuenta con aspectos positivos y también áreas de mejora; los aspectos positivos que sobresalen son que el 91 \% del capital humano se siente preparado para adaptarse al rápido crecimiento y expansión de la empresa; un 74 \% considera tener la experiencia y las habilidades necesarias para ayudar a la organización en su etapa de crecimiento hacia nuevos mercados. En lo que respecta al ambiente laboral, el $65 \%$ considera que disfruta de un ambiente óptimo para el desempeño de las funciones. Así mismo, con un $91 \%$, el capital humano contestó que sería sencillo adaptarse a nuevos procedimientos dentro de la empresa.

Por otra parte, las áreas de oportunidad que el capital humano considera son definir las funciones que realizan en la empresa de manera comprensible, sencilla y por escrito al momento de ser contratados; es necesario agregar que no existe un manual de procedimientos en donde el trabajador pueda apoyarse o guiarse para realizar sus actividades. Así mismo, es indudable que el trabajador no recibe la capacitación adecuada para el desempeño de sus funciones, lo cual puede generar problemas o crear una barrera para el crecimiento.

En cuanto a la existencia de algún departamento que pudiera estar perjudicando o deteniendo el proceso de crecimiento, los resultados revelan que en primer lugar se halla el departamento de logística; su principal motivo es la falta de planeación en el proceso de entrega de pedidos hacia los clientes, presentando retrasos en los plazos establecidos de envío de productos.

En cuanto a las mejoras que la empresa debería realizar para su crecimiento hacia nuevos mercados, en opinión del capital humano, se debe implementar estrategias para hacer más eficientes los procesos, diseñar un manual de organización y manuales de procedimientos, crear y difundir programas de capacitación y desarrollo profesional y personal, fortalecer el programa de comunicación interna, los programas de compensación, beneficios y servicios, y con ello mejorar la calidad del trabajo. Por lo tanto, la empresa cuenta con el capital humano comprometido a mejorar las áreas de oportunidad y con ello alcanzar los objetivos organizacionales. 


\section{REFERENCIAS}

Alonso, R. (2012). Capital Humano. Gary Becker y su trabajo en el concepto de capital humano. Recuperado de http:// teoria-del-capital-humano.blogspot.mx/2012/o1/gary-becker-y-su-trabajo-en-el-concepto.html

Álvarez, J., Fraiz, A. y Del Río, M. (2013). Implantación de un sistema de gestión de la calidad: beneficios percibidos. Universidad del Zulia Maracaibo, Venezuela, Revista Venezolana de Gerencia,18(63), 379-407. Recuperado de http:// www.redalyc.org/articulo.oa?id=29028476002

Benavides, L. (2012). Medición, valoración y determinación del impacto del capital intelectual en la generación de valor en la empresa tendencias. Universidad de Nariño, Colombia. Revista de la Facultad de Ciencias Económicas y Administrativas, XIII (1)1, 100-115. Recuperado de https://dialnet.unirioja.es/servlet/articulo?codigo=4023998

Calderón, J., y Mousalli, G. (2012). Capital humano: Elemento de diferenciación entre las organizaciones. Universidad de los Andes Mérida, Venezuela, Actualidad Contable Faces, 15(24), 05-18. Recuperado de http://www.redalyc.org/ articulo.oa? $\mathrm{id}=25722697002$

Chiavenato I. (2007). El capital Humano de las organizaciones (8va. ed.). McGraw-Hill.

Cirer, J. (2008). Capital humano y desarrollo económico, una compleja relación. Estudios de Economía Aplicada, 26(3). Recuperado de http://www.redalyc.org/articulo.oa?id=301/30113182005

Deeb, C. (2013). ¿Qué le ocurre a una organización con una mala administración? La Voz de Houston. Recuperado de http://pyme.lavoztx.com/qu-le-ocurre-una-organizacin-con-una-mala-administracin-13402.html

Del Canto, E. (2011). Gestión del capital humano, competencias y sociedad del conocimiento. Observatorio Laboral Revista Venezolana, 4(8). Recuperado de http://www.redalyc.org/articulo.oa?id=2 190/219022148006

Domínguez, M. (2008). Factores determinantes en la gestión de recursos humanos en empresas de servicios que incorporan de manera sistemática nuevas tecnologías. Un estudio de caso en la comunidad valenciana. Revista de la Escuela de Negocios de la Universidad del Norte. Pensamiento \& gestión, 1 (24). Recuperado de http://rcientificas. uninorte.edu.co/index.php/pensamiento/article/view/3503/4923

Falestchi, G. (2013). Crecimiento y desarrollo en las organizaciones de Kiruv. Recuperado de http://nleresources. com/es/2013/08/crecimiento-y-desarrollo-en-las-organizaciones-de-kiruv/

Hernández, R., Fernández, C. y Baptista, P. (2006). Metodología de La Investigación. Recuperado de https://es.scribd. $\mathrm{com} /$ doc/38757804/Metodologia-de-La-Investigacion-Hernandez-Fernandez-Batista-4ta-Edicion

Huertas, J. (2012). El papel de la gestión del capital humano en la creación de una ventaja competitiva. Recuperado de http://gestionestrategicachjuliohuertas.blogspot.mx/2012/10/el-papel-de-la-gestion-del-capital.html

Instituto Nacional de Estadística, Geografía e Informática (INEGI). (2015). Encuesta nacional sobre productividad y competitividad de las micro, pequeñas y medianas empresas. México: Instituto Nacional de Estadística, Geografía e Informática. Recuperado de https://www.inegi.org.mx/programas/enaproce/2015/

Kerlinger, F. y Lee, H. (2002). Investigación del comportamiento (4ta. ed.). McGraw-Hill Interamericana.

Lanzagorta, J. (25 de octubre 2011). Cierran 80 \% de las pymes en México por la falta de previsión. El Economista. Recuperado de https://www.eleconomista.com.mx/sectorfinanciero/Cierran-80-de-las-Pymes-en-Mexico-por-faltade-prevision--20111025-0125.html

Martínez, J. (2005). Administración y organizaciones. Su desarrollo evolutivo y las propuestas para el nuevo siglo. Universidad de Medellín, Colombia, Semestre Económico, 8(16), 67-97. Recuperado de http://www.redalyc.org/ pdf/ 1650/ 165013663004.pdf 
Montilla, O. (2003). El capital humano en la valoración de empresas. Revista de la Facultad de Ciencias Económicas: Investigación y Reflexión, XI(1). Recuperado de http://www.redalyc.org/articulo.oa?id=909/90911107

Muratalla, G., Gaona, H., García, J. y Guzmán, L. (2016). El capital humano: factor determinante para incrementar la productividad en la empacadora de aguacate de exportación en Michoacán. Revista Vinculatega de la Universidad Autónoma de Nuevo León, 1(2). Recuperado de http://www.web.facpya.uanl.mx/vinculategica/Revistas/R2/3327$3349 \% 20-\% 20$ El\%20Capital\%20Humano\%2OFactor\%20Determinante\%20Para\%20Incrementar\%20La\%20 Productividad\%20En\%20La\%20Empacadora\%20De\%20Aguacate\%20De\%20Exportacion\%20En\%20Michoacan. pdf\#page $=1$

Navarro, I. (2005). Capital Humano: Su definición y alcances en el desarrollo local y regional. Archivos Analíticos de Políticas Educativas, 13(1),1-36. Recuperado de https://www.redalyc.org/pdf/2750/275020513035.pdf

Organización Internacional del Trabajo. (2016). Mejore su negocio: El recurso humano y la productividad (1ra. ed.). Recuperado de https://www.ilo.org/wcmsp5/groups/public/---ed_emp/---emp_ent/---ifp_seed/documents/ instructionalmaterial/wcms_553925.pdf

Pérez, J.(2012). Adaptabilidad: ¿somos adaptables? Recuperado de http://depersonasyrecursos.blogspot.mx/2012/09/ adaptabilidad-somos adaptables.html

Ramírez, M., Texis, M. y Aguilar, J. (2014). El papel del capital humano y el aprendizaje en las microempresas de base social en Baja California. Estudios Fronterizos, Revista de Ciencias Sociales y Humanidades, 15 (29). Recuperado de http://redalyc.org/articulo.oa?id=53031359007

Reyes, L. y García, Y. (2009). Procedimiento para el perfeccionamiento de la gestión del capital humano. Centro de Información y Gestión Tecnológica de Santiago de Cuba, Ciencias Holguín, XV(2), 1-14. Recuperado de http://www. redalyc.org/articulo.oa?id=181517990003

Villalobos Monroy, G. y Pedroza Flores, R. (2009). Perspectiva de la teoría del capital humano acerca de la relación entre educación y desarrollo económico. Universidad Autónoma del Estado de México, Toluca, México, Tiempo de Educar, 10(20), 273-306. Recuperado de: https://www.redalyc.org/pdf/311/31112987002.pdf 